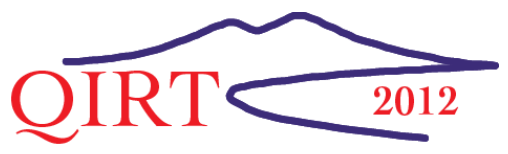

\title{
Infrared Monitoring of Surface Temperature Distributions on Electron Beam Targets in Applications for Fusion Technology
}

by R.E. Nygren*, D.L. Youchison* and T.J. Lutz*

*Sandia National Laboratories ${ }^{1}$, Albuquerque, New Mexico, USA renygre@sandia.gov

\begin{abstract}
Plasma facing components (PFCS) for future fusion reactors and for existing experiments around the world receive intense heat loads that vary in time and in space. Sandia National Laboratories performs high heat flux testing in our Plasma Materials Test Facility to simulate these heat loads. We monitor the targets with IR cameras and obtain data from pyrometers, thermocouples and coolant calorimetry to determine their thermal performance. We present examples from targets with carbon-fiber-composite tiles, beryllium tiles, copper heat sinks, helium-cooled refractory PFCs and liquid lithium.
\end{abstract}

\section{Introduction}

Fusion is an advanced power source. Its primary market would be to supplant fission power over a transition in the future. In a fusion reactor, two hydrogen isotopes, deuterium (D) and tritium (T), combine to form helium. This is the same fusion reaction in a hydrogen bomb and in the sun and other stars, but our terrestrial approach confines the fusion plasma using magnetic fields. Two physics experiments, the Toroidal Fusion Test Reactor that operated at the Princeton Plasma Physics Laboratory and the Joint European Torus or JET at Culham Laboratory have produced D/T fusion reactions during relatively brief "shots".

Each fusion reaction produces $17.6 \mathrm{MeV}$ of energy with $80 \%$ imparted to a neutron and $20 \%$ imparted to the helium nucleus. The neutron penetrates into the structure. The electrically charged helium nucleus follows a path dictated by the magnetic field and distributes most of its energy within the plasma. This energy, plus additional power injected to control the fusion plasma, is eventually collected on the surfaces surrounding the plasma either as radiated power from the plasma or as power from particles that strike the surfaces. The use of infrared thermography to observe the distribution of this power in confinement experiments and in related high heat flux testing is the subject of this paper.

\subsection{Fusion Plasma Facing Components}

The protective tiles of plasma facing components (PFCs) in magnetic confinement experiments cover the surfaces that face the plasma. During shots of 2-10 s (sometimes longer at lower powers in current experiments) the plasma heats these PFCs. Then they cool by conduction and radiation between shots. To increase their power-handling capability, two fusion experiments, Tore Supra and the Large Helical Device, have installed watercooled PFCs. An example from work on Tore Supra is shown later. The next generation of devices that will run with higher power and have longer shots (tens of seconds to hours) must have actively-cooled PFCs.

Construction has now begun on, ITER, the world's first engineering fusion reactor.[1] This project, sited at France's nuclear research center, the Commissariat à l'Energie Atomique (CEA), is the world's

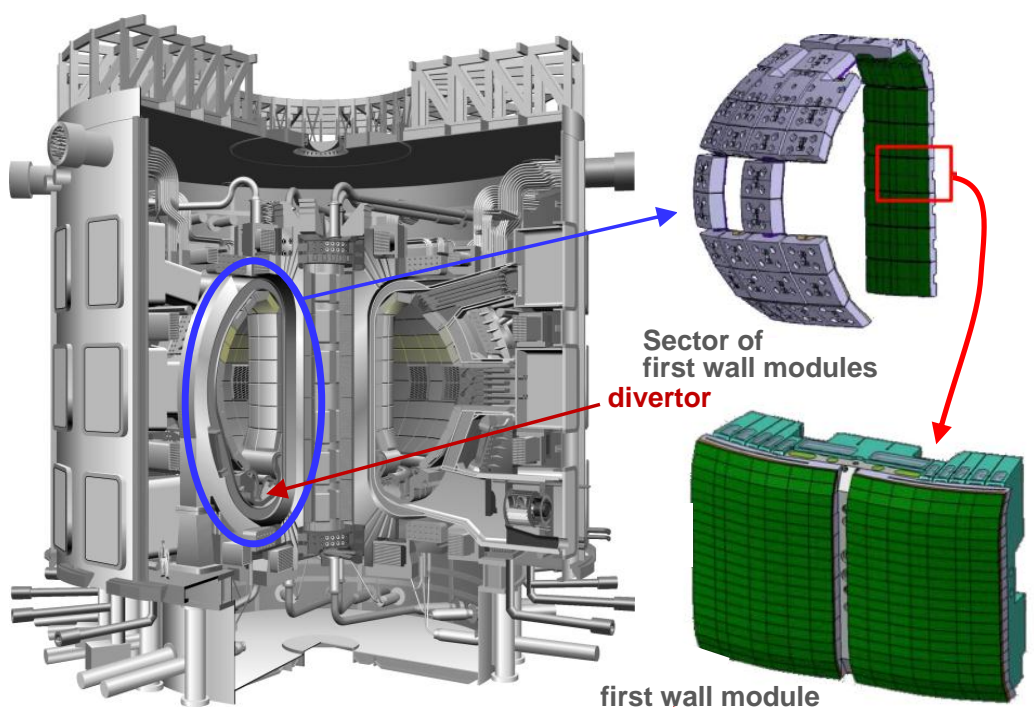

Fig. 1. Cross section of ITER with separate view of first wall (top), first wall sector (right) and Enhanced Heat Load Module (bottom) largest international science collaboration. Figure 1 is a cutaway view of ITER. Most of the interior surface open to the donut-shaped plasma is what we call the first wall. The figure shows a sector of the first wall and a first-wall-shield module with "fingers" that run laterally and have tiles shown in green. The smaller chamber below the main volume that contains the plasma is called the divertor. Portions of the divertor typically receive a higher steady state heat loads than

\footnotetext{
${ }^{1}$ Sandia is a multiprogram laboratory operated by Sandia Corporation, a Lockheed Martin Company, for the United States Department of Energy's National Nuclear Security Administration under contract DE-AC04-94AL85000. Work at the Princeton Plasma Physics Laboratory is supported by USDOE Contract DE-AC02-09CH11466.
} 
the first wall. Experiments with divertors have a magnetic configuration that directs the ions and electrons at the edge of the plasma, and the associated power flow, into the divertor where most of the power deposits along a narrow circumferential stripe ${ }^{2}$ that we call the "strike point." The estimated peak heat load on the divertor during steady state operation is $\sim 10 \mathrm{MW} / \mathrm{m}^{2}$. On the "enhanced heat load" portions of the first wall (for which Sandia has led the design R\&D for the US and coordinated the design effort for the international ITER team), the anticipated heat loads are 1-5 $\mathrm{MW} / \mathrm{m}^{2}$ and less on the rest. Water at $120^{\circ} \mathrm{C}$ is the coolant for the PFCs in ITER. A temperature above $100{ }^{\circ} \mathrm{C}$ is needed to reduce condensables (e.g., moisture) that would otherwise be retained on the walls and become impurities in the plasma.

Future magnetic fusion reactors will use $\mathrm{He}$ and liquid metal or liquid salt as high-temperature coolants and working fluids that have good efficiency for power conversion. The sections that follow begin with a discussion of the distribution of power on PFCs and some applications of IR thermography in magnetic confinement experiments. Subsequent sections draw upon examples in high heat flux testing that are part of the R\&D to develop PFCs.
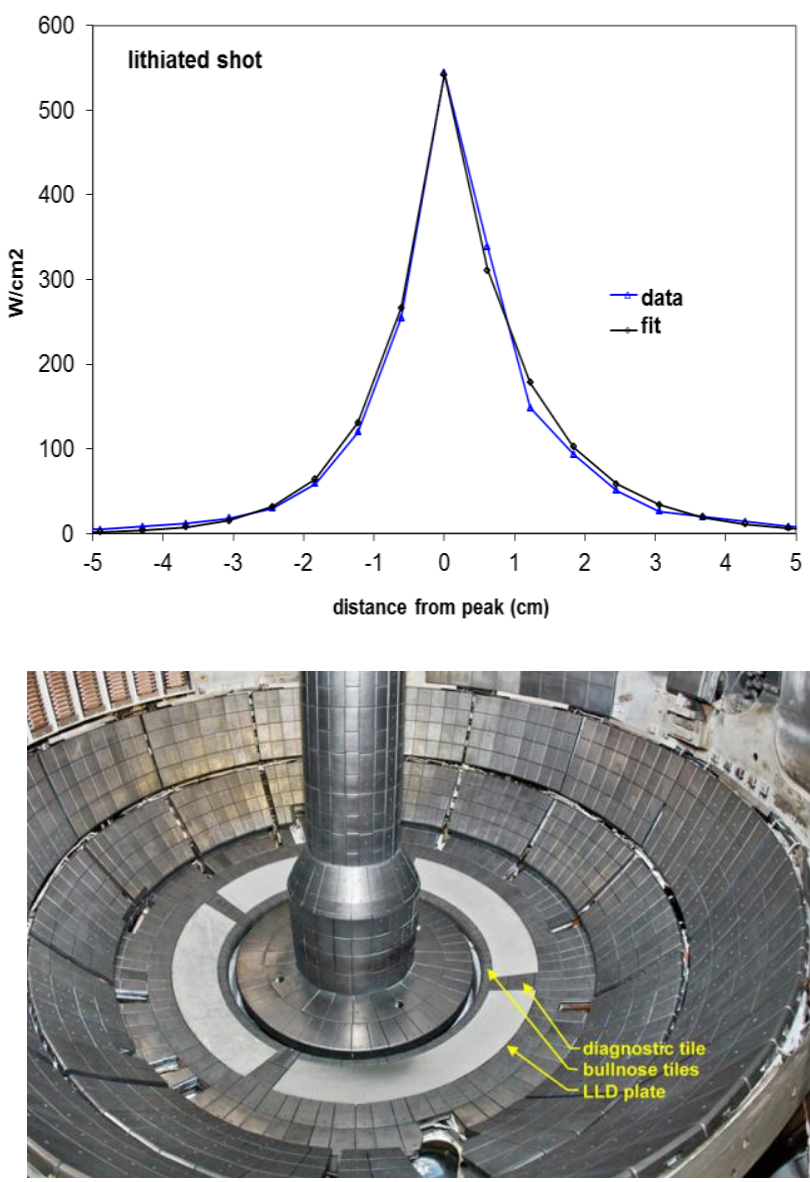

Fig. 2. Temperature profile at the divertor strike point (top) and Liquid Lithium Divertor plates in NSTX (bottom).

\subsection{Power on Fusion Plasma Facing Components (PFCs)}

Fusion researchers using IR cameras in magnetic confinement experiments are typically trying to determine the heat load on PFCs. This paper uses examples from NSTX, the National Spherical Torus Experiment, at the Princeton Plasma Physics Laboratory (PPPL) in Princeton, New Jersey. A strong program on the beneficial effects of lithium at the edge of the plasma motivated the installation of a divertor dominated by surfaces of liquid lithium to replace large portions which had tiles of ATJ graphite.[2,3] Figure 2 shows the heat load on the portion of the divertor near the strike point, where the maximum is $\sim 5.5 \mathrm{MW} / \mathrm{m}^{2}\left(550 \mathrm{~W} / \mathrm{cm}^{2}\right)$ and the material in the divertor is lithium. These data were extracted from IR measurements when lithium had been deposited on the ATJ graphite in the divertor. A later section of this paper discusses IR measurements of the divertor in NSTX when the graphite was replaced by a liquid lithium divertor, as shown in the bottom of Figure 2.

Measuring the shape of the temperature profile at the strike point is a primary objective because it reveals an important feature of the plasma. A broad peak in profile of temperature (and deposited power) spreads the power over a wider circumferential stripe and the peak temperature is lower than for a sharper profile. We characterize the narrowness of the profile with the term "power scrape-off length" or $\lambda_{q}{ }^{3}$, a very basic and important quantity for designing PFCs. The narrower the power scrape-off length, the higher are the peak temperatures and thermal stresses for a given power level. Hard engineering limits for the reactor materials, such as tungsten-based armor for the walls, will set the acceptable level for power production through the allowable stresses. Moreover, the power-scrape-off length arises from selfbalancing processes in the plasma and cannot be manipulated independently.

Scaling with size is also important. A larger volume of plasma would better insulate the core plasma from the walls and be attractive for the economics of a reactor. But this presents a problem for power onto PFCs. The fusion power (proportional to plasma volume) scales with size cubed, whereas the area of the PFCs that receive $20 \%$ of this power scales only with the size squared.

Power handling is a critical area for fusion. As yet, our ability to predict the power scrape-off lengths in future devices is quite limited due to the complex physics and heat transport in the of the edge plasma.[4] Workable solutions for PFCs must be compatible with the plasma, through choices of materials, and integrated with a tritium-breeding blanket needed to provide fuel by breeding tritium from lithium.

${ }^{2}$ The magnetic configuration of a diverted plasma produces both an inner and an outer strike point, typically with differing power loads, but the basic explanation here applies to either.

${ }^{3}$ The curves on either sides of the peak are usually approximated with functions of the form, $\mathbf{q}=\mathbf{q}_{0} \exp \left(-\mathbf{x} / \lambda_{q}\right)$, where $\mathbf{q}$ is the heat flux (or power density), $\mathbf{x}$ is the distance away from the peak, and $\lambda_{q}$ is the power-scrape-off length noted above. The characteristic values of $\lambda$ differ on the two sides of the profile because the quantities differ in the processes such as collisions with neutral atoms and impurities from the walls that affect the temperatures and densities of the ions and electrons in the edge plasma. 


\subsection{High Heat Flux Testing of PFCs}

For 25 years we have extensively utilized IR thermography in our high heat flux testing to develop PFCs and other high performance heat sinks at Sandia's Plasma Materials Test Facility (PMTF). $[5,6]$ In the PMTF the rastored electron beams of our 60 kW system, EB60, or our $1.2 \mathrm{MW}$ dual beam system, EB1200, can heat actively-cooled targets in large vacuum chambers. (See Figure 3.)

We monitor the distributions of the surface temperatures of these targets with infrared (IR) cameras during testing and use the IR data from both scanning and digital focal plane arrays, along with data from various other diagnostics such as pyrometers, thermocouples and coolant calorimetry, to determine the thermal performance of these targets. For example, we perform thermal cycling

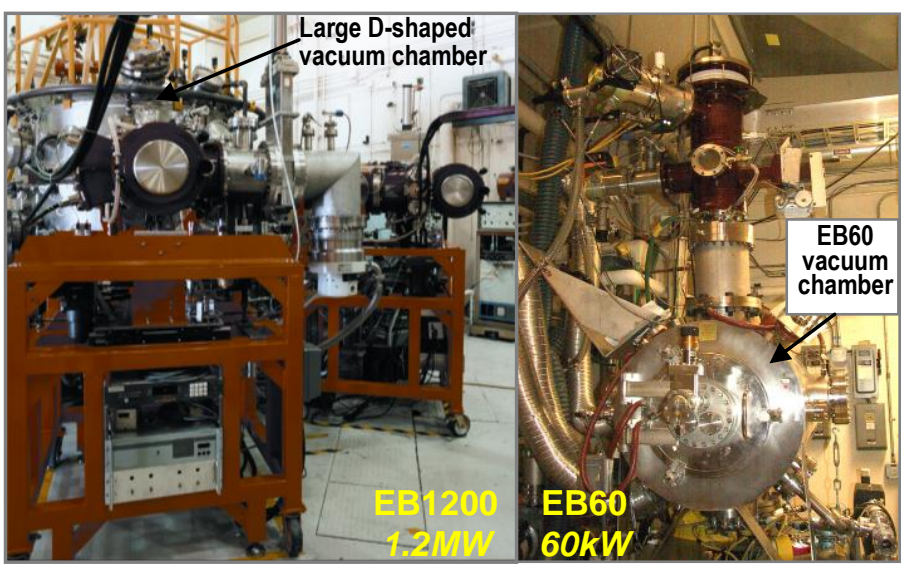

Fig. 3. Photos of electron beam test stands, EB1200 with two beamlines (left) and EB60 with e-gun overhead (right).

tests with heat loads in the range of $5-20 \mathrm{MW} / \mathrm{m}^{2}$ to demonstrate the robustness of heat sinks with armor joined to high performance actively-cooled substrates. In other tests, we see whether a target can survive a specified maximum heat load or how intensely we can heat the target before some precursor to failure is detected.

The IR cameras are mounted externally on view ports with ZnSe windows on the vacuum chambers for EB60 and EB1200. We use FLIR SC4000 cameras and also have used Inframetrics Model 760, and before 1996, Model 600 cameras. Typically, we also have data from two color pyrometers and can use these data for an independent measurement of temperature that permits us to extract a calibration factor that accounts for the emissivity of a surface but also includes the optics of our viewing system. We compare the experimental results with 3-D finite element models of heat transfer and stress.

\subsection{Quantitative IR thermography}

The paper is an overview of various types of testing and the role of IR, but does not go into details about the significant quantitative measures that underlie the results of testing at Sandia, as in the following processes. Author Youchison has developed computer software, SandIR, that directly reads the camera output and displays colorized IR frames. We can independently assign emissivities to various regions of the frame with flexibility in creating the shapes and we can plot the surface temperatures in various ways with a suite of tools. We use these data for real time feedback in controlling the e-beams, for example to halt a test when a region exceeds an allowable temperature. Our calibration procedures give us true temperatures in addition to temperature distributions, and we can do pixel by pixel calibration of emissivities that include the optics of our system. We compare these data with extensive thermal modeling. In many of our test campaigns, the quantitative IR data and true temperatures are important both for the management of the test, for example in stopping a test before failure of the target can produce excessive evaporation of material, and for comparison with our thermal models to determine the thermal performance of a PFC. The paper presents some examples. Reference 6 gives a detailed description of the processes we utilized in testing of ITER mockups described later in Section 2.1. Since the targets reflect a fraction of the power from the e-beams, the PMTF has extensive diagnostics for calorimetry to complement the IR data and produce reliable values of the absorbed power, which is an essential parameter in our evaluations of thermal performance.

An underlying theme in this paper is the issues associated with the emissivities of the surfaces tested. Among the problems encountered are that emissivities change during the test due to modifications of the surfaces such as oxidation, damage, melting or vaporization and deposited layers. Also, non-uniform heat conduction can make interpretation of data difficult.

\section{Uses of IR imaging in developing PFCs for fusion}

We believe IR thermography is an indispensable tool in high heat flux testing. For the tests in PMTF at Sandia, we record IR data with the emissivity set at unity with the tools in the software. We also correct individual frames used for evaluation in real time by adjusting the emissivity with calibrations based on data from pyrometers and from thermocouples embedded in the targets. Reference 7 gives a detailed explanation of our testing in PMTF and our procedures, using the evaluation of thermal cycling tests done for the ITER Project as an example. The targets for these tests (called ITER First Wall Quality Mockups) have features identical to a section of a first wall finger, shown in green in Figure 1, but with a shorter heated length and covered with three beryllium tiles. 

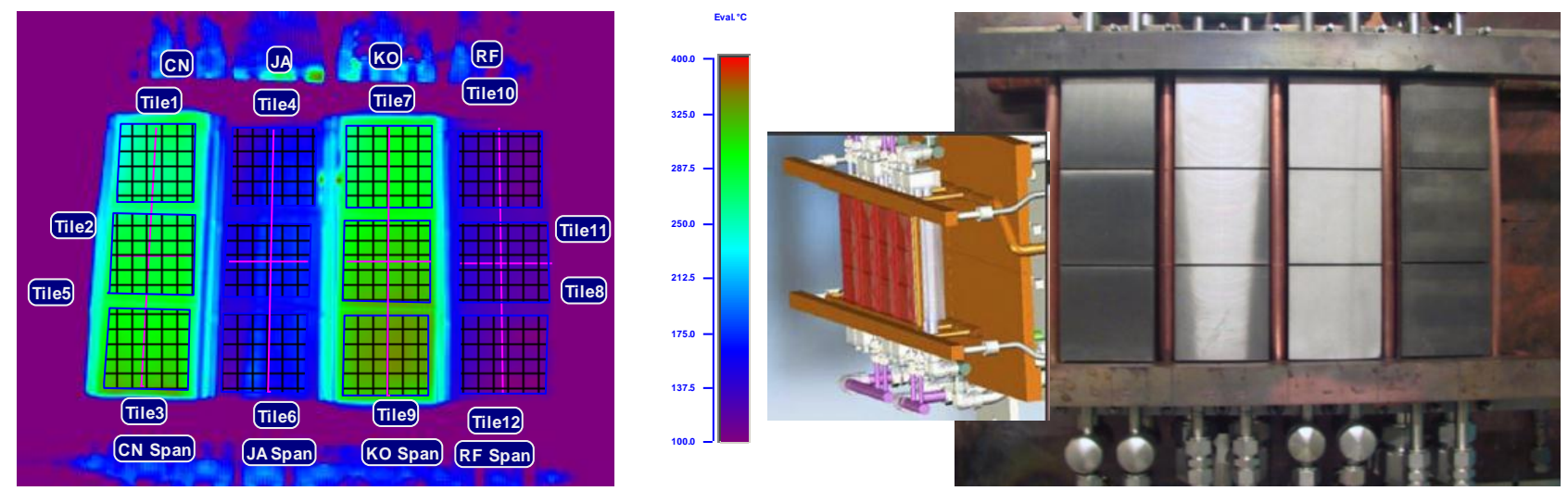

Fig. 4. Colorized IR image (left) of thermal cycling test of ITER first wall quality mockups, left to right, from China $(C N)$, Japan (JA), Korea (KO) and Russia (RF); drawing and photo (right) show mounting on EB1200 door.
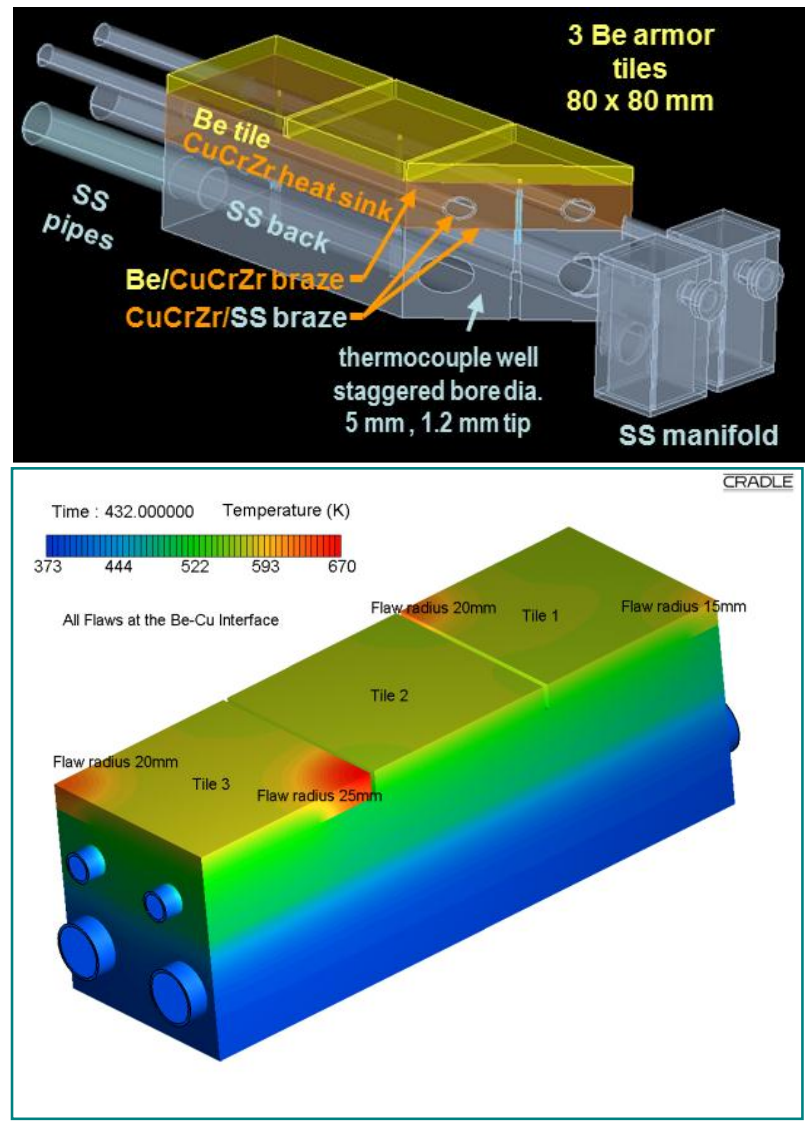

Fig. 5. CAD sketch (top) and thermal model (bottom) of an ITER FWQM with flaws or cracks at corners of tiles.

\subsection{Tests of ITER First Wall Quality Mockups (FWQMs)}

Figure 4 shows a colorized IR image of ITER FWQMs built by the US and EU in thermal cycling tests in PMTF with the Chinese and Korean mockups heating while the Japanese and Russian mockups cool. In these tests done to 12,000 thermal cycles, each of the two electron beams was directed alternately at a pair of mockups to give thermal cycles of $30 \mathrm{~s}$ on and $30 \mathrm{~s}$ off.[7] These mockups have three beryllium tiles joined by brazing or hot isostatic pressing to a water-cooled CuCrZr heat sink that is joined to a water-cooled 316 stainless steel backing. In each tile, a thermocouple protrudes from the back side of the mockup to just below the plasma-facing surface.

Likely sites for failure are the joints between ITER's beryllium tiles and the $\mathrm{CuCrZr}$ heat sink. An increase in the surface temperature near a corner or edge can indicate the beginning of failure. Figure 5 shows an early thermal analysis of the temperature distributions on an ITER FWQM with faults in the joints of several sizes.[8] We did not see such failures in our testing, but our approach with IR thermography would reveal such features, and has in the past on other types of PFC targets.

Some testing of ITER FWQMs is also done elsewhere in the world at a facility with a radiant heat source in which the graphite heaters close to the heated surface preclude the possibility of IR thermography. Where IR thermography would easily detect the non-uniform temperature distribution over a crack at the corner of a tile, the central thermocouple provides only a very coarse indication of a problem in the joint. We at Sandia feel this is an inferior approach to testing the ITER FWQMs.

\subsection{Testing for Critical Heat Flux}

Our testing has also included studies of Critical Heat Flux or CHF (burnout) in many water-cooled PFCs. Burnout is a well-known and well-studied phenomenon, e.g., cooling for fission reactors and steam generators. Typically in these cases, water is flowing up or down around or inside a tube. The event leading to burnout occurs when the previously-sub-cooled water boils and vapour forms in the entire annular zone adjacent to the heated wall. Defining the margin against burnout is an important criterion in designs for PFCs and requires good data on CHF.

In our PFCs, heating occurs from only one side, and an observable precursor precedes burnout and heat flows around a vapor blanket localized just below the heated surface. The example here is from testing of an early version of the water-cooled heat sinks for the ITER first wall. The same set of samples were tested at labs in the EU, the US and Japan in a "round-robin" experiment to compare the results among the labs.[8] 


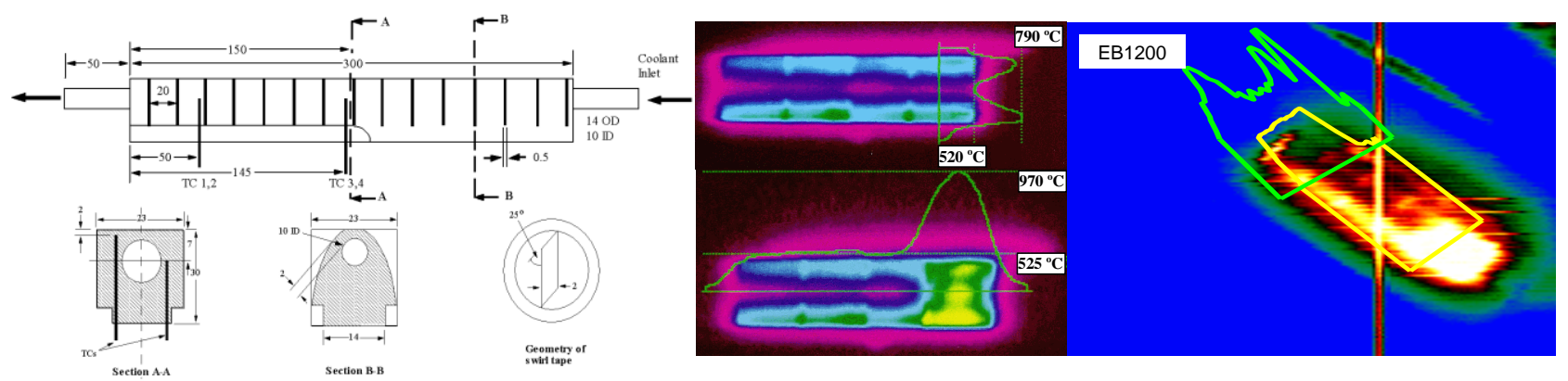

Fig. 6. Colorized IR frames from CHF tests in the French facility FE-200 (middle two) and in EB1200 in PMTF (right) on a single-channel water-cooled heat sink shown at the left.

The targets were single-channel water-cooled heat sinks of Glidcop Al-25 with twisted tape inserts. The twisted tape increases the margin against CHF. With $100^{\circ} \mathrm{C}$ water at $12 \mathrm{~m} / \mathrm{s}$ the CHF was $27 \mathrm{MW} / \mathrm{m}^{2}$. The main point for this paper is that the shift in the distribution of surface temperatures, observed with IR cameras, permitted researchers to anticipate the burnout and stop the test before burnout occurred. The middle set of frames in Figure 6 compares the pattern for stable cooling (top) with one (bottom) when vapor starts to insulate the area just below the heated surface. This portion of the surface becomes hotter and appears yellow in the bottom frame. The thermograph of the right side shows a similar test in EB1200. The temperature profile (green line) across a line in the upper left portion of the sample shows peaks on the outside, similar to the condition in the upper middle thermograph, and the white spot in the lower right is the area where the temperature has risen due to the vapour blanket that precedes $\mathrm{CHF}$.

\subsection{Braze flaws}

Our next example shows the use of IR thermography to distinguish braze flaws as Sandia was building a watercooled modular PFC for the Tore Supra tokamak at CEA. The PFCs here comprised a set of shaped copper tubes each with a set of tiles of highly oriented pyrolytic graphite brazed to the copper tubes.[9] Operation of Tore Supra has provided the primary experience with water-cooled PFCs in fusion prior to the development of PFCs for ITER.

Figure 7 shows a composite of colorized IR frames in a time series of 2, 4, 6, 8, 10, 12, 16, 20 and $30 \mathrm{~s}$ for one of the hot water tests used to identify flawed brazes. For these tests, individual "fingers" of the limiter started at room temperature and a flow of hot water was rapidly introduced into the copper pipe. The temperatures of well brazed tiles rose rapidly, and those with flawed brazes less rapidly. We used the tests to identify tiles with sufficiently poor thermal bonding to require remaking of the part. The IR data in Figure 7 shows no tiles lagging in temperature compared with their neighbors, and confirmed that rebrazing of tube $1 \mathrm{e}$ had been successful.

In this case the combination of the IR data and thermal modeling gave a very good indication of the type of the braze flaws that could be identified in testing, and whether the flaws would be acceptable for the application. And these quantitative criteria were used to accept or reject tubes after the step when tiles were brazed. The plots (from Ref. 9) show the applied heat load (top) and heat flux from the tube wall into the cooling water (bottom) and its relation to the type of braze flaw. Due to the high conductivities of pyrolytic graphite and copper, fairly large flaws were acceptable.

The approach of hot water testing with IR thermography to evaluate joined parts was used subsequently at SATIR (Station d'Acquisition et Traitement InfraRouge) facility[10] at CEA and is also now being used in a
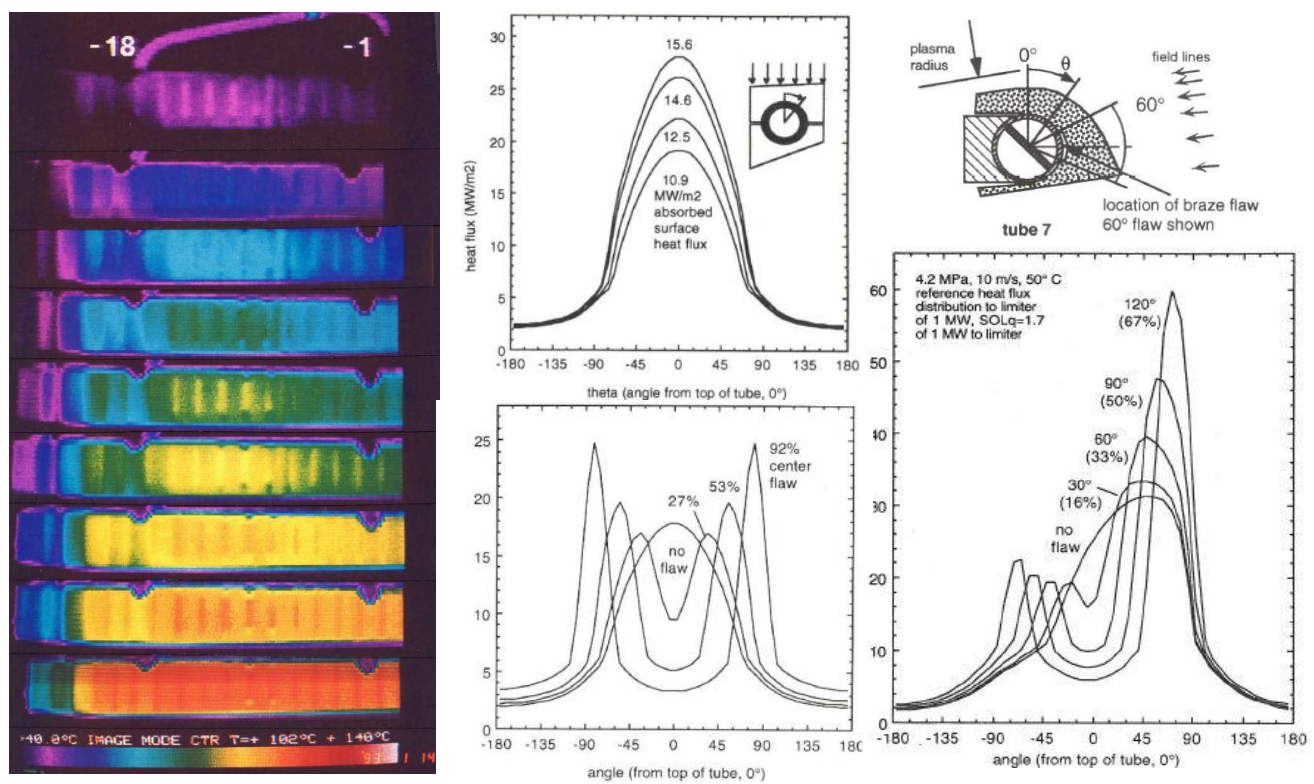

Fig. 7. Combined colorized IR images (left) of a Tore Supra Phase III Limiter tube 1e during a hot water test; plots show applied heat load to tube $3 e$ (top middle) and heat flux into water versus position on tube and similar plot (right) for tube 7 at leading edge of limiter. 


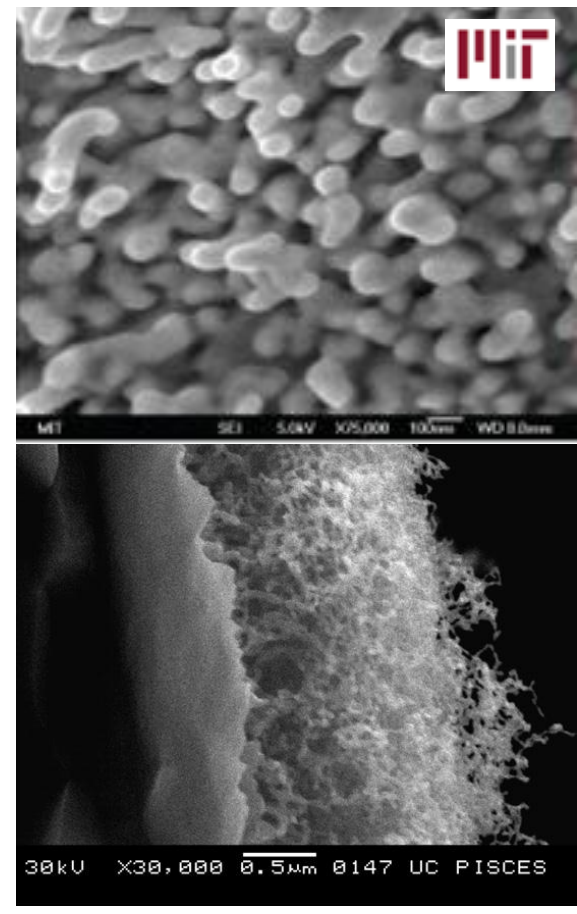

Fig. 8. Tungsten fuzz grown in PISCES (bottom) and tungsten tendrils found in Alcator C-Mod (top), courtesy of MIT's Plasma Fusion Science Center and of the Jacobs School of Mechanical and Aerospace Engineering at UCSD.

more sophisticated form by Japanese researchers at the Japan Atomic Energy Agency to evaluate the quality of brazes for graphite tiles for the ITER divertor.[11]

\section{Issues with Emissivity}

In the applications for fusion, the targets we monitored in the PMTF with IR cameras (and pyrometers) typically are in vacuum and we observe through windows, e.g., zinc selenide for our broad range IR cameras. The determination of absolute temperature, from which we could determine the absorbed power density at locations of interest, requires some calibration for the optics of the system as well as a value of emissivity for the surface being observed. Coating of windows occurs but the measures to deal with this are fairly straightforward. Unless direct calibration of our system is available, e.g., where we receive unambiguous data from two-color pyrometers, this information is obtained through experience with the systems and perhaps some accompanying thermal modeling. A more complex issue is that our conditions in testing can modify the surfaces being observed. The whole area of plasma-surface interactions is a very active area of investigation in fusion ${ }^{4}$.

\subsection{Surface features}

Ideally, surface preparation is important and high-emissivity finishes provide better IR data. However, a frequently encountered problem with fusion PFCS is surface layers with unknown emissivity and thickness and an imperfect thermal bond to the substrate. In fusion experiments, this can happen when material eroded from PFCs by the plasma, through sputtering or vaporization, is redeposited to form surface layers. In devices with carbon tiles and hydrogen plasmas, which has been the norm for many past and present magnetic fusion experiments, carbon typically redeposits with hydrogen to form a kind of plastic coating.[12] But other types of deposits including "sooty" deposits with "forests" of carbon structure with very low average density have also been found.[13] In these cases, it is obvious that the IR results are not consistent with the expected behavior of a dense well characterized carbon surface, as is true with poorly attached surface layers, but the problem of interpretation of data remains. Also, in our experiments for fusion, the IR results typically are part of a much larger set of measurements related to the deposited power and conditions at the plasma edge, as well as thermal modeling of the components.

For future fusion reactors, tungsten is the leading choice for the plasma facing material. Some current plasma experiments operate with refractory metal walls, including the ITER-like wall experiment in JET, the ASDEX-Upgrade tokamak at the Max Planck Institute for Plasma Physics in Garching, Germany, and the ALCATOR C-Mod tokamak at the Massachusetts Institute of Technology's Plasma Fusion Science Center.

Recently, a surprising surface morphology of tiny tendrils of tungsten was observed for the first time in a tokamak, specifically in C-Mod.[14] Similar forms have been grown in linear plasma devices.[15,16] Figure 8 shows micrographs of the features from surfaces of the probe in C-MOD and from surfaces modified in the PISCES linear plasma device at the University of California, San Diego (UCSD). The thickness of the tendrils found in C-MOD are 50$100 \mathrm{~nm}$, which is thicker than the 20-30 nm tendrils in the tungsten fuzz grown in the linear plasma source PISCES at UCSD. Optically these surfaces appear black.

In these cases, the limitation in interpretation is the lack of knowledge of such characteristics as thickness, conductance, adherence, and emissivity. We also face similar limitations for some intentionally applied coatings

\subsection{Coatings}

Many fusion experiments coat PFC surfaces in-situ with boron because it reduces the retention of hydrogen on the walls. So the surfaces observed with IR are really boron rather than carbon or another underlying wall material. Also, the potential for depositing beryllium coatings in-situ on a PFC with beryllium tiles has been investigated as a repair technique for ITER (and for JET). Vaporization, sublimation (of carbon) and melting also can modify surfaces and change their emissivities, as can evolution of the surface due to damage as noted above. The following three examples, taken from our research, show cases with (1) melting of metal, (2) sublimation of carbon, and (3) a beryllium coating with unknown thermal properties.

\footnotetext{
${ }^{4}$ Work in this area is reported, among other places, in the International Conference on Plasma Surface Interactions that has been published by Elsevier, Inc. The $20^{\text {th }}$ conference in the series was in Aachen, Germany in June 2012
} 
Melting of material affected the IR thermography in high heat flux testing of early US-built ITER divertor mockups performed at the Japan Atomic Energy Agency.[17] The mockups had tungsten rods embedded in a water-cooled copper heat sink. The IR data were referenced to a calibrated pyrometer. A puzzling feature observed in the pyrometer traces for mockup PW-14 was oscillations at $\sim 5 \mathrm{~Hz}$ in the temperatures for several shots, as shown in the plot in Figure 9.

In this mockup, the technique for embedding the tungsten rods in the copper alloy host included placing them in an Inconel honeycomb that maintained their alignment in the joining step done by hot isostatic pressing. During the test, some of the honeycomb material, although well below the top surface of the tunsten rods, melted and coated the sides of the adjacent tungsten rods.

We postulated that, in subsequent shots, this melted material wetted the sides and top of the rods. Recalibration of the pyrometer showed much higher apparent temperatures, which suggests that the emissivity of the surface had changed. We concluded that the oscillations arose from the dynamic combination of evaporative cooling from the surface and resupply of the surface coating from melted material moving up the side of the rods, rather than from some effect in the signal processing, because this effect occurred after the melting and was not observed in tests of two other tungsten rod mockups.

Resolution was also an issue for the IR data on the 3.2-mm-diameter tungsten rods. The high signal (white-out) on a hot rod would tend to bleed into the pixels of an adjacent rod. This issue also affect the next case discussed.
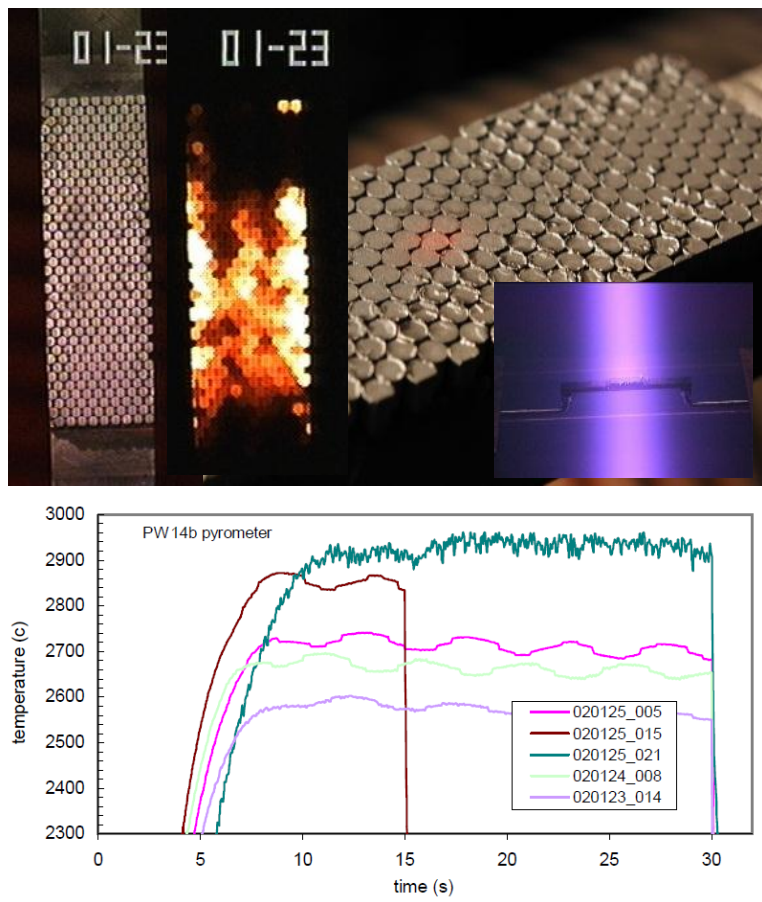

Fig. 9. Photos of $\mathrm{CuCrZr}$ heat sink armored with rods; video frame (second from left) shows heated sample with many "white hot" rods; at upper right is post-test photo; inset shown ion beam (blue light) during test.

For applications in a planned fusion device called TPX (not built) and then for KSTAR, a tokamak in Korea, we tested various types of carbon fiber composites (CFCs).[18] Our test used an approach previously developed to test various types of graphite. In EBTS (predecessor to EB60), we heated an area of $10 \times 10 \mathrm{~mm}$ within samples that were $25.4 \times 25.4 \times 10 \mathrm{~mm}$. The thermal expansion of the inner area produces thermal stresses that would tend to fracture graphites but not the tougher CFCs. In the tests of these CFCs, we heated the targets for times from 1.5 to $6 \mathrm{~s}$ at absorbed heat loads from $6-110 \mathrm{MW} / \mathrm{m}^{2}$. Figure 10 is a composite of photos of several CFC targets. Each CFC target had a single spring-loaded thermocouple that reached up from the back to the center of the sample. We estimated the
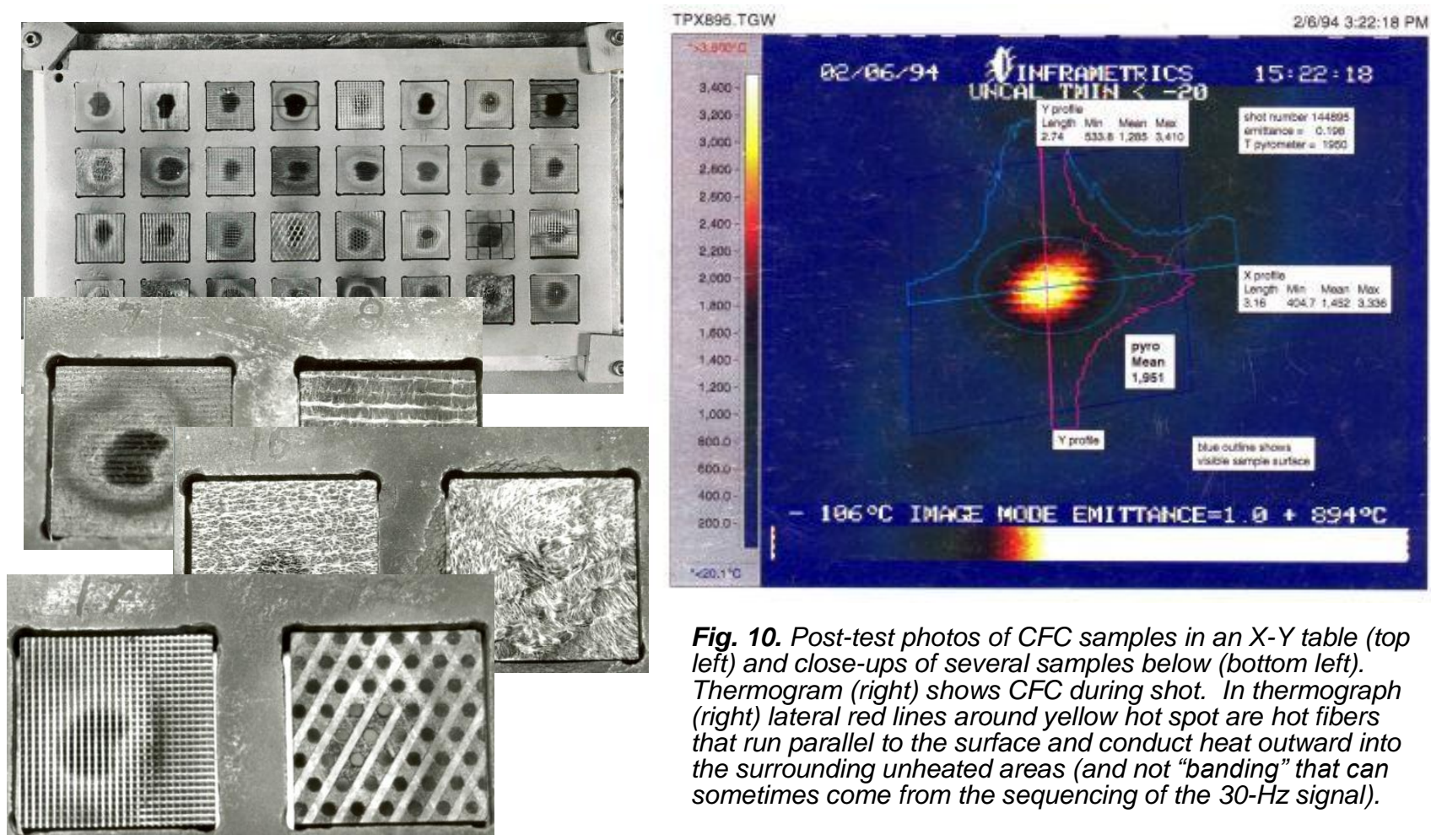

Fig. 10. Post-test photos of CFC samples in an X-Y table (top left) and close-ups of several samples below (bottom left). Thermogram (right) shows CFC during shot. In thermograph (right) lateral red lines around yellow hot spot are hot fibers that run parallel to the surface and conduct heat outward into the surrounding unheated areas (and not "banding" that can sometimes come from the sequencing of the $30-\mathrm{Hz}$ signal). 
absorbed heat from the temperature gain during the shot and after the temperature had equilibrated within the target. A complication for the IR was the high temperature for materials in which fiber tows ran parallel to the heated surface. These fibers had limited transverse conductance to adjacent material and reached high temperatures that saturated the IR camera, and at the higher heat loads significant sublimation occurred from these fibers and the adjacent binder.

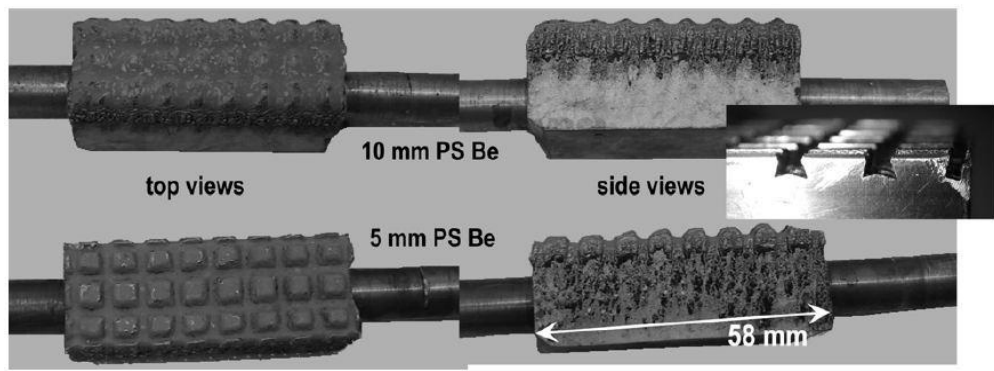

Fig. 11. Top and side views of mockups with 5-mm or 10-mm coatings of plasma sprayed beryllium. Inset photo shows the tesselated base.

The third example is from testing at Sandia done in collaboration with Los Alamos National Laboratory where heat sinks with plasma-sprayed beryllium armor deposited on tessellated CuCrZr, were fabricated.[19,20] Figure 11 shows the two types of mockups.

In this case the density and thermal conductance of the deposited surface were unknown. We estimated the thermal properties of the deposited coating as follows. We assumed that the plasma sprayed materials were not fully dense but that, within the coating, the beryllium itself had the conductivity of pure beryllium. Using the surface temperature from IR data with a simple thermal model, we estimated the density of the deposited coating to be about $40 \%$. An attendant issue was changing emissivity due to oxidation of the beryllium, which also was observed and accounted for in our tests of the ITER FWQMs.

\section{Liquid Surface PFCs}

Concepts for fusion reactors with liquid walls have been proposed.[21,22] The primary advantage would be that the continually replenished plasma facing surfaces would not accumulate damage from neutrons that would degrade the properties of solid walls, nor would there be the problem of melting. A second motivation, specifically for liquid lithium, is that liquid lithium at the edge of the plasma appears to improve plasma performance. Research with lithium has been a strong thrust in the research for the NSTX Team.[2,3] In collaboration with PPPL, Sandia built the hardware for the Liquid Lithium Divertor, shown previously in Figure 2, that was installed for the 2010 campaign in NSTX.[23] The LLD did not have flowing liquid lithium but rather a set of four plates with embedded electrical heaters that could melt a layer of lithium on the surface of the plates. Each copper plate had a thin stainless steel layer with a porous coating of flame sprayed molybdenum to keep the molten lithium in place during operation.

An important objective of the experimental campaign with the LLD was to confirm that one of the beneficial effects of lithium in the plasma edge is to increase the power scrape-off length, $\lambda_{\mathrm{q}}$, compared to operation without lithium. For this experiment, the NSTX Team had developed a dense array of Langmuir probes in the divertor[24] and a fast (2 $\mathrm{kHz})$ dual-wavelength IR camera (4-6 and 7-10 $\mu \mathrm{m})$ with a split beam with which to eliminate emissivity as a variable.[25] Interpretation of the data was challenging because of the unknown properties of the lithium surface.

The effective thermal conductivity of the mixed layer of lithium and molybdenum could be estimated, but the emissivity of the lithium surface also depended on the degree of surface contamination. Lithium is a reactive metal that reacts with water, oxygen, carbon and nitrogen and scavenges these within the vacuum present in NSTX. An initially shiny surface of nearly pure lithium will turn dull and become gray and eventually white when reacting with water or oxygen and black when reacting with carbon or nitrogen. Allain and co-workers at Purdue University had studied the surface chemistry of lithium deposited on graphite.[26] Sandia is planning a definitive experiment with Allain and coworkers at Purdue University to measure the emissivities of lithium over a range of temperatures with lithium in the solid or liquid state and with various degrees of surface contamination.

Extensive thermal modeling was done before the installation of the LLD and continues. The output was estimates of the temperature of the lithium for various heat loads for comparison with IR data during operation.[27] Figure 12 shows results. Modeling has continued with the goal of explaining some of the results observed with the LLD. Much of the previous evaluation (by others) of the thermal behavior of the plate assumed a simple semi-infinite solid with the rise in temperature proportional to the square root of time. But this is inaccurate for the LLD, where copper's high conductivity permits heat to diffuse rapidly both inward from the heated surface and laterally away from the peak heating.

In the plot on the upper left of Figure 12 (next page), by $0.5 \mathrm{~s}$ after the shot starts (by $4800.5 \mathrm{~s}$ on the axis) heat has reached the back of the Cu plate (bottom of LLD) and the Li surface is over $140^{\circ}$ higher than the underlying Cu (top surface). After this time the plate does not behave like a semi-infinite solid because the thermal gradient through the plate has been established, and after $4800.5 \mathrm{~s}$ the temperature rises more slowly and roughly linearly with time. At the end of the 5-s shot, the $\mathrm{Li}$ has risen to $875 \mathrm{~K}$ from $\sim 650 \mathrm{~K}$. When the shot ends, the heat load ceases and the temperatures near the top surface drop and those at the bottom increase as the plate attains its average temperature. Explanations involving broadening of the heat load due to evaporative cooling and lithium in the plasma edge have been proposed to explain the rollover of the temperature curve in the first second of the shot. However, the authors will show next that this feature can easily be explained as the simple and expected result of heat conduction in the LLD itself. 

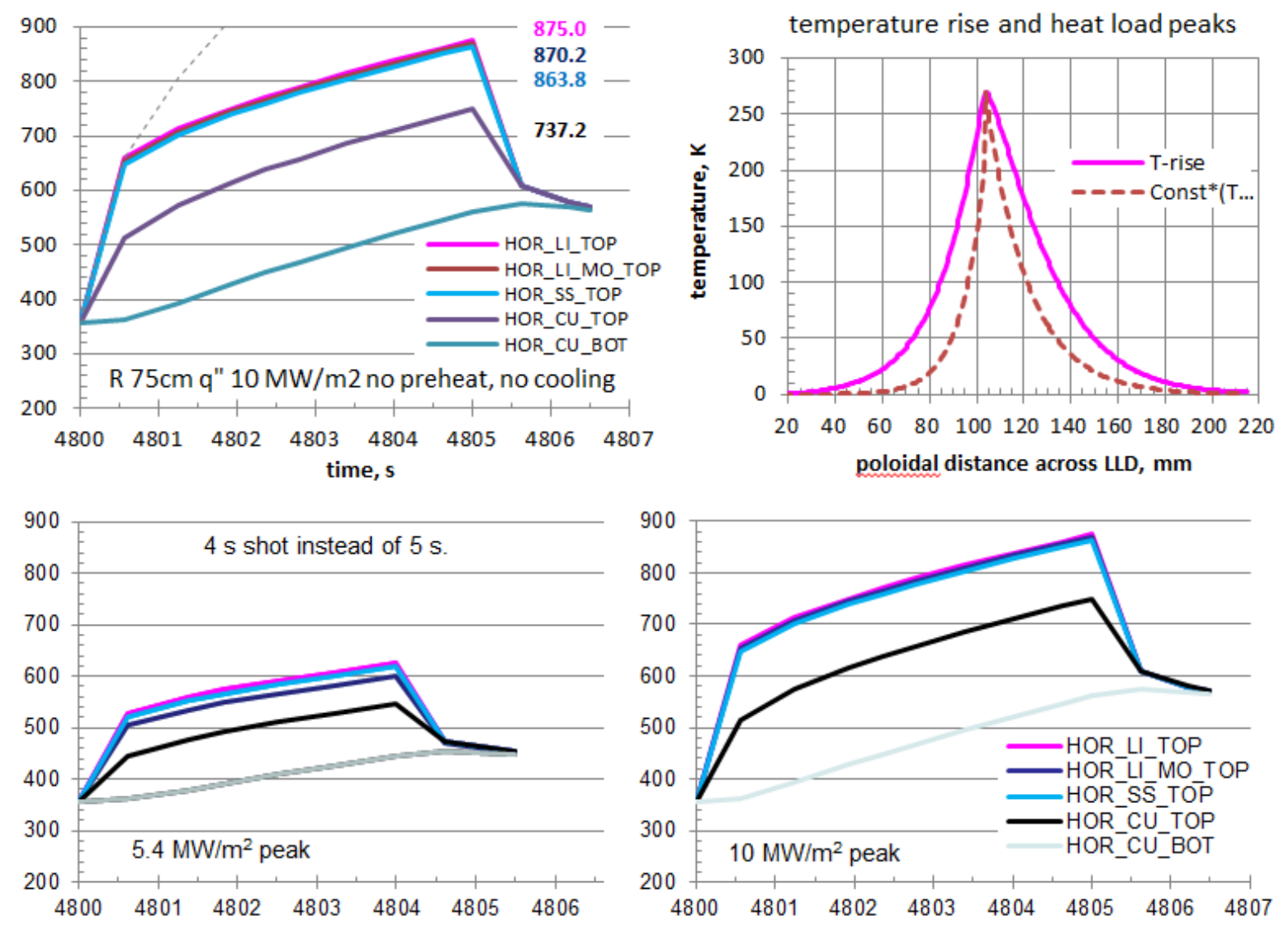

Fig. 12. Plots from a 3-D thermal model that provided an alternate explanation of "thermal broadening" due to lithium at the plasma edge.

The rise in the temperature of the $\mathrm{Li}$ is proportional to the heat load, as can be verified by the plots at the bottom of Figure 12. After $4 \mathrm{~s}$ at10 $\mathrm{MW} / \mathrm{m}^{2}$, the rise is $465^{\circ}$ and at $5.4 \mathrm{MW} / \mathrm{m}^{2}$ it is $262^{\circ}$. In each case the rise in temperature divided by the heat load is 46.6 degrees per $\mathrm{MW} / \mathrm{m}^{2}$. So evaporative cooling is not a significant factor. And, lateral conduction of heat is important as the initial peaked heat load diffuses into the LLD. If the loading is changed from a peaked load to a uniform load of $5.4 \mathrm{MW} / \mathrm{m}^{2}$, so there is no lateral heat conduction, the temperature rises to over $800^{\circ}$.

Finally, the plot at the upper right shows the shape of the peak heat load superimposed with a normalized distribution of temperature from the end of the shot, when the peak has broadened by about a factor of two. The temperature rises during the shot, and the peak progressively broadens due to lateral heat conduction in the plate. This broadening is greatest at the end of the shot when the temperatures are highest. This is also the time when the IR data are the most likely to be selected for evaluation.

\section{Closing Comments}

This paper is a retrospective summary using various examples of the application of infrared thermography in R\&D supporting the development of plasma facing components for the realization of fusion energy as a power source. A general observation from our experience in high heat flux testing at Sandia National Laboratories is that infrared thermography is indispensable both qualitatively and quantitatively. An example of qualitative application is to judge the uniformity of heat loads through the observed distribution of temperatures on the heated surfaces of our targets. An example of quantitative application is when we develop results with values of true temperature based on high temperature calibrations of emissivity. This information is important in understanding the performance of the heat sinks we test. The IR data are one part of a suite of information that is needed for this purpose. Careful measurements of the absorbed power and actual heated area of the targets are also extremely important, and related information includes coolant calorimetry, data from pyrometers and from thermocouples in the targets and the results from thermal modeling.

Facilities for high heat flux testing using electron beams have the flexibility to simulate complex heat loads that can vary spatially, as in the peaked heat loads on divertors, and in time, for example as in the simulation of rapid transient heat loads or thermal cycles with ramp up and ramp down features. We believe that for high heat flux testing of plasma facing components for fusion applications, use of electron beam facilities with appropriate power and diagnostics is the most effective way to evaluate the thermal performance of PFCs in advance of service in fusion experiments. 


\section{REFERENCES}

[1] http://www.iter.org (general and technical information about the ITER Project)

[2] M. Ono, M.G. Bell, R. Kaita, H.W. Kugel, J-W. Ahn, J.P. Allain, et al., Recent Progress of NSTX lithium program and opportunities for magnetic fusion research, Fusion Engineering \& Design or FED (2011, in press)

[3] H.W. Kugel, M.G. Bell, H. Schneider, J.P. Allain, R.E. Bell, R. Kaita, et al., Lithium coatings on NSTX plasma facing components and its effects on boundary control, core plasma performance, and operation, FED 85 (2010) p865-873

[4] D.G. Whyte, B. LaBombard, J.W. Hughes, B. Lipschultz, J. Terry, D. Brunnen et al., Constraining the divertor heat width in ITER, $20^{\text {th }}$ Int. Conf. on Plasma Surface Interactions, Germany, June 2012, to be published

[5] JM McDonald, RE Nygren, DL Youchison, T.J. Lutz, High Power Electron Beam Technology, EBEAM2004 International Conference on High-Power Electron Beam Technology, Reno, Nevada, Oct. 2004 (presentation)

[6] R.E. Nygren, D.L. Youchison, T.J. Lutz, J.M. McDonald, M.A. Ulrickson, Sandia's Plasma Materials Test Facility: high heat flux electron beam experiments for the ITER First Wall Program, EBEAM2010 - International Conference on High-Power Electron Beam Technology, Reno, Nevada, Oct. 2010

[7] T.J. Tanaka, R.E. Nygren, D.L. Youchison, and M.A. Ulrickson, Considerations in Testing of ITER First Wall Mockups, FED 82, 15-24 (2007) p1884-1892

[8] M. Rödig, I. Bobin-Vastra, S. Cox, F. Escourbiac, A. Gervash, A. Kapoustina, W. Kuehnlein, N. Litounovsky, M. Merola, R. Nygren, D.L. Youchison, Testing of Actively Cooled Mockups in several high Heat Flux Facilities An International Round Robin Test, FED 75-79 (2005) 303-306

[9] R. Nygren, T. Lutz, J. Miller, R. McGrath and D. Guilhem, Assessing Braze Quality in the Actively Cooled Tore Supra Phase III Outboard Pump Limiter, FED 28 (1995) p113

[10] M. Missirlain, H. Traxler, J. Boscary, A Durocher, F. Escorbiac, J. Schlosser et al., Infrared thermography inspection methods applied to the target elements of W7-X divertor, FED 82/15-24 (2007) p1747-55

[11] Y. Seki, K. Ezato, S. Suzuki, K. Yokoyama, M. Enoeda, S. Mori, Non-destructive examination with infrared thermography system for ITER divertor components, FED 85/7-9 (2010) p1451-54

[12] W.L. Hsu, R.A. Causey, Pumping of hydrogen during plasma-graphite interaction, J. Vacuum Science \& Tech. 5/4 (1987) p2768-72

[13] J.P. Coad, B. Farmery, Redeposition effects on the target plates of the JET tokamak, Vacuum 45/4 (1994) p435-39

[14] G.M. Wright, D. Brunner, M.J. Baldwin, B. Labombard, B. Lipschultz, J.L. Terry, D.G. Whyte, Tungsten nanotendril growth in the Alcator C-Mod divertor, Nuclear Fusion 52 (2012) doi:10.1088/0029-5515/52/4/042003

[15] R.P. Doerner, M.J. Baldwin. P.C. Stangeby, An equilibrium model for tungsten fuzz in an eroding plasma environment, Nuclear Fusion, 51/4 (2011) 043001 doi:10.1088/0029-5515/51/4/043001

[16] S. Kajita, W. Sakaguchi, N. Ohno, N. Yoshida, T. Saeki, Formation process of tungsten nanostructure by the exposure to helium plasma under fusion relevant plasma conditions, Nuclear Fusion 49/9 (2009) 095005 doi:10.1088/0029-5515/49/9/095005

[17] R. E. Nygren, D. L. Youchison, J. S. O'Dell, High Heat Flux Tests on Heat Sinks Armored with Tungsten Rods, FED 49-50 (2000) 303

[18] R.E. Nygren, D.L. Youchison, J. Kim, K.H. Im, K.S. Kim, and H.C. Park, High Heat Flux Tests of Carbon Composites for KSTAR and NSTX, (SOFE2002) IEEE Symp. On Fusion Eng. ISBN D-7803-7073-2

[19] R.E. Nygren, D.L. Youchison, K.J. Hollis, Features of plasma sprayed beryllium armor for the ITER first wall, JNM 367-37- (2007) p1325-29

[20] K.J. Hollis, B.D. Bartram, M. Rödig, in: Int. Thermal Spray Conf., 2005, Basel 2.4.5.2005

[21] R.E. Nygren, T.D. Rognlien, M.E. Rensink, S.S. Smolentsev, M.Z. Youssef, M.E. Sawan, B.J. Merrill, C. Eberle, P.J. Fogarty, B.E. Nelson, et al., A Fusion Reactor Design with a Liquid First Wall and Divertor, FED Special Issue 72, 1-3 (2004) 223-244

[22] Articles in the special issue of FED 72 1-326 (2004)

[23] R.E. Nygren, G.R. McKee, J. A. Fordham, S.A. Lewis, H. Kugel, R.A. Ellis, M. E. Viola, J.S. O’Dell, Preparation of the Liquid Lithium Divertor Plates for NSTX, J. Nucl. Mat. 417/1-3 (2011) p592-96

[24] J. Kallman, M.A. Jaworski, R. Kaita, H. Kugel, High density Langmuir probe array for NSTX scrape-off layer measurements under lithiated divertor conditions, Rev. of Sci. Instruments 81/10 (2010) 10E117 (2010) http://dx.doi.org/10.1063/1.3494381 (3 pages)

[25] J.H. Nichols, A.G. McLean, R. Maingi, J.W. Ahn, A.L. Roquemore, B.C. Stratton, R. Kaita, H.W. Kugel, K. Gan, M. Benjamin, Design and Deployment of a Wide-Angle Two-Color Infrared Camera with Optical Relay on NSTX, Bulletin of the American Physical Soc., 56/16 (2011) BAPS.2011.DPP.PP9.70

[26] J.P. Allain, D.L. Rokusek, S.S. Harilal, M. Nieto-Perez, C.H. Skinner, H.W. Kugel, B. Heim, R. Kaita, R. Majeski, Experimental studies of based surface chemistry for fusion plasma-facing materials applications, J. Nucl. Mater. 390-391 (2009) p942

[27] R. E. Nygren, H. C. Harjes, P. Wakeland, R. Ellis, H. W. Kugel, R. Kaita, L. Berzak, L. Zakharov, B. Ehrhart, Thermal Control of the Liquid Lithium Divertor for NSTX, FED 84/7-11 (2009) p1438-1441; also (poster \& presentation) R. Nygren, M. Sceiford, H. Kugel, J. Kallman, A. McLean, Thermal Modeling of the Surface Temperatures on the Liquid Lithium Divertor in NSTX, $2^{\text {nd }}$ Int. Symp. on Lithium Applications, Princeton Plasma Physics Laboratory (April 2011) 